\title{
Mitochondrial dysfunction and Alzheimer's disease: prospects for therapeutic intervention
}

\author{
Ji Woong Lim ${ }^{1}$, Jiyoun Lee ${ }^{2, *} \mathcal{E}$ Ae Nim Pae ${ }^{1,3, *}$ \\ ${ }^{1}$ Convergence Research Center for Diagnosis, Treatment and Care System of Dementia, Korea Institute of Science and Technology, Seoul \\ 02792, ${ }^{2}$ Department of Global Medical Science, Sungshin University, Seoul 01133, ${ }^{3}$ Division of Bio-Medical Science \& Technology, KIST \\ School, Korea University of Science and Technology, Seoul 02792, Korea
}

\begin{abstract}
Alzheimer's disease (AD) is a multifactorial neurodegenerative disease and has become a major socioeconomic issue in many developed countries. Currently available therapeutic agents for AD provide only symptomatic treatments, mainly because the complete mechanism of the AD pathogenesis is still unclear. Although several different hypotheses have been proposed, mitochondrial dysfunction has gathered interest because of its profound effect on brain bioenergetics and neuronal survival in the pathophysiology of AD. Various therapeutic agents targeting the mitochondrial pathways associated with AD have been developed over the past decade. Although most of these agents are still early in the clinical development process, they are used to restore mitochondrial function, which provides an alternative therapeutic strategy that is likely to slow the progression of the disease. In this mini review, we will survey the AD-related mitochondrial pathways and their small-molecule modulators that have therapeutic potential. We will focus on recently reported examples, and also overview the current challenges and future perspectives of ongoing research. [BMB Reports 2020; 53(1): 47-55]
\end{abstract}

\section{INTRODUCTION}

Alzheimer's disease (AD) is a chronic neurodegenerative disorder, characterized by the progressive loss of memory and neuronal degeneration. The prevalence of $A D$ is high among aged individuals, and the incidence rate for $A D$ increases exponentially after age 60 . With the extended human average lifespan, $A D$ is becoming an increasingly widespread health issue and tremendous research efforts have been made to

${ }^{*}$ Corresponding authors. Ae Nim Pae, Tel: +82-2-958-5185; Fax: +82-2-958-6999; E-mail: anpae@kist.re.kr; Jiyoun Lee, Tel: +82-2920-7281; Fax: +82-2-920-2027; E-mail: jlee@sungshin.ac.kr

https://doi.org/10.5483/BMBRep.2020.53.1.279

Received 19 October 2019

Keywords: Alzheimer's disease, Mitochondrial dysfunction, Mitochondrial therapeutics, Neurodegenerative diseases design and develop novel drugs that can efficiently stop the progress of the disease (1). Given that aging is accompanied by a time-dependent progressive deterioration of multiple pathways in the cells, high energy-demanding organs such as the brain would greatly suffer from the age-dependent alteration in bioenergetics and redox homeostasis, leading to neuronal impairment and eventually to neurodegeneration. Notably, brain hypometabolism and oxidative stress are the prominent events in $\mathrm{AD}$, which undoubtedly involve metabolic pathways and electron-transport systems in the mitochondria (2-4). Indeed, mitochondrial dysfunction is an early feature of $\mathrm{AD}$ and appears to play a significant role in its pathogenesis (4-6).

Mitochondria are multi-functional organelles responsible for a wide range of functions, including adenosine triphosphate (ATP) production, metabolism, and stress responses. Given the diverse roles of mitochondria, mitochondrial dysfunction has been linked to a variety of diseases, such as diabetes, cardiovascular disease, cancer, and neurodegenerative diseases. In particular, the central nervous system requires approximately $20 \%$ of the body's total basal oxygen consumption to support neuronal energy expenditure; therefore, neurons with impaired mitochondrial function suffer from ATP depletion, oxidative stress, and eventually cell death (7). Brain hypometabolism and increased oxidative stress were observed in brains from $A D$ transgenic mice and $A D$ patients even before the appearance of senile plaques and neurofibrillary tangle (NFT) $(2,4)$. These two main hallmark proteins of $A D$ also have a direct effect on mitochondrial function, causing the impairment of mitochondrial membrane potential, the reduction of ATP production, and the acceleration of ROS generation in the brain $(8,9)$. Now many researchers have reached the hypothesis that restoring normal mitochondrial function may increase neuronal survival and potentially provide a therapeutic strategy to reverse the disease course of $\mathrm{AD}$ (10-14).

Currently available therapeutic agents approved for the treatment of $A D$ provide only symptomatic relief, but $A D$ therapies that can stop or slow the progression of the disease are urgently needed (15). In this context, compounds that can rescue dysfunctional mitochondria might generate a novel 
therapy for AD. To this end, compounds targeting the pathways associated with mitochondrial function have been developed over the past decades, and some of these compounds are currently in clinical trials for the treatment of AD. In this review, we will overview the AD-related mitochondrial dysfunction and potential therapeutic strategies that have been reported recently. We will also describe small-molecule functional modulators that target mitochondria (Table 1) and discuss their advantages and future challenges in the development of novel therapeutic agents for the treatment of AD.

\section{MITOCHONDRIAL PROTEIN-QUALITY CONTROL}

Healthy mitochondria maintain protein homeostasis by regulating protein folding, degradation, and fission and fusion processes to repair and remove damaged mitochondrial proteins, the overall processes of which are also termed protein-quality control (16). Mitochondrial dysfunction disrupts the protein-quality control processes, leading to the accumulation of the damaged and misfolded proteins inside mitochondria, eventually promoting the pathogenesis of various neurodegenerative diseases. The pathways and processes associated with protein-quality control are regulated at multiple levels, from proteolytic degradation at the molecular level to apoptosis at the cellular level; at the center of the stage, mitochondrial dynamics and mitochondrial protein degradation, also known as mitophagy, are critically involved (17). Whereas the eventual downstream effects of the defective mitochondrial quality control are the accumulation of neurotoxic, misfolded proteins associated with various neurodegenerative diseases, including AD, Parkinson's disease (PD), and Huntington's disease (HD) (18), in this section we will focus on the pathways and small-molecule modulators involved in the AD pathology.

Mitochondrial dynamics: Dynamin-related protein 1 (Drp1) Mitochondria are highly dynamic organelles undergoing constant cycles of fusion and fission, forming the mitochondrial network, which is important for the control of the distribution, shape, and size of mitochondria, and also crucial for the regulation of cell-death pathways (19). The fine balance between the fusion and fission process is maintained by mitochondrial membrane proteins such as optic atrophy proteins (OPA) and mitofusins (MFN). Mutations in these proteins lead to a defective mitochondrial network, causing a slew of issues including impaired bioenergetics, disrupted protein-quality control, and mitochondrial proliferation (20). Many studies have indicated that the defective mitochondrial network is one of the major culprits in the pathogenesis of neurodegenerative diseases including Parkinson's disease and Alzheimer's Disease (20). Specifically, the mitochondrial fission protein, dynamin-related protein (Drp1), exacerbates $A D$ pathogenesis by inducing excessive mitochondrial fragmentation and abnormal mitochondrial dynamics upon interacting with $A \beta$ and tau proteins (21-23). Increased Drp1 activity also increases ROS production but abates ATP production, causing oxidative damage and synaptic dysfunction in $A D$ neurons. Several studies also have found increased levels of Drp1 in postmortem AD brains as well as animal AD models (24-26), suggesting that the inhibition of Drp1 may generate therapeutic effects in AD.

Over the past decade, small-molecule Drp1 inhibitors have been developed and tested for their therapeutic efficacies in AD animal models. The most widely studied inhibitor, mdivi-1 is a quinazolinone-based cell-permeable Drp1 inhibitor which inhibited mitochondrial division in yeast and mammalian cells (27). More importantly, studies have found that the treatment with mdivi-1 demonstrated neuroprotective effects in transgenic $A D$ mice by reducing excessive mitochondrial fragmentation $(28,29)$. In addition to mdivi-1, P110, a heptapeptide (DLLPRGT) derived from the sequences of Drp1, demonstrated similar protective effects by blocking mitochondrial fission and restoring ROS-induced mitochondrial dysfunction in neuronal cells $(30,31)$. Several research groups have developed small-molecule inhibitors with more potent Drp1 inhibitory effect by chemical library screening and structural optimization. Numadate et al. reported PAQ-22 (3-(4-chloro-3-methoxyphenyl)2-thioxoquinazoline-4-one) (32); Mallat et al. identified two effective compounds containing the $1 \mathrm{H}$-pyrrole-2-carboxamide scaffold (33). More recently, Kuruva et al. designed a novel Drp1 inhibitor named DDQ (diethyl (3,4-dihydroxyphenethylamino) (quinolin-4-yl)methylphosphonate), based on the molecular docking study of the $A \beta$ and Drp1 protein complex. DDQ inhibited the $A \beta$ and Drp1 interaction, reduced cellular levels of $A \beta$ oligomers, and improved mitochondrial function in cell-based models of AD (34). Modulating the mitochondrial fusion and fission by using a small-molecule Drp1 inhibitor such as mdivi-1 is a novel strategy that can potentially generate a disease-modifying drug. However, considering that these inhibitors have been tested in only cells and a few selected animal models and a potential off-target effect was also reported (35), they may still have a long way to go before clinical development.

\section{Mitophagy: PINK1 (PTEN-induced kinase 1)}

PINK1 is a mitochondrial serine/threonine kinase located in the mitochondrial intermembrane space, and its association with early-onset PD is widely recognized (36). PINK1 detects damaged mitochondria, initiates mitophagy by recruiting cytosolic protein partner Parkin, and removes damaged mitochondria (37). Although PINK1 mutation is considered to be the hallmark of early-onset PD, several recent studies have found that PINK1 signaling is also associated with Alzheimer's disease $(38,39)$. According to the study reported by Du et al., PINK1 is downregulated in the brains of AD patients as well as in the transgenic mouse model, and restoring the PINK1 activity in the transgenic mice improved cognitive function 


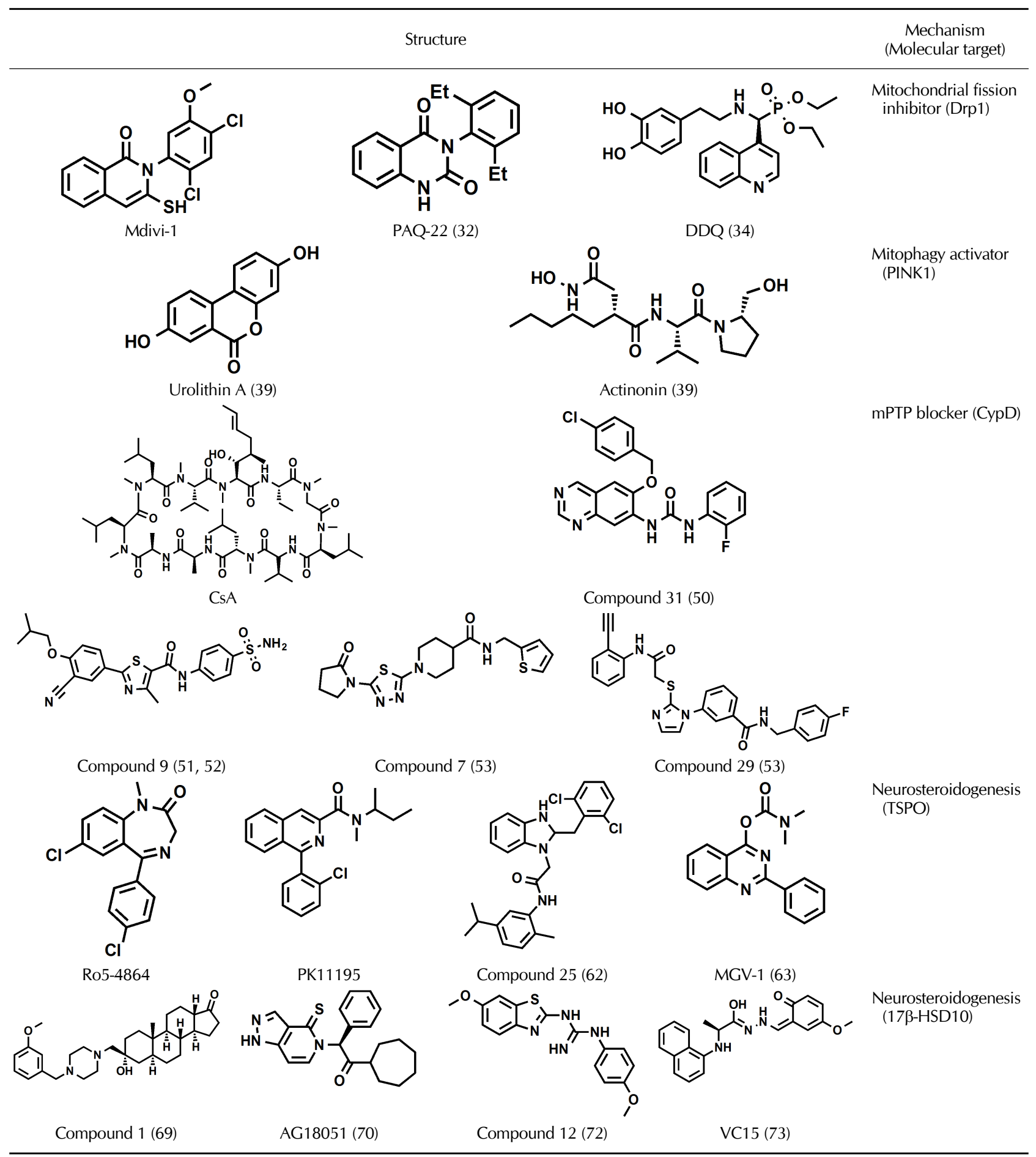




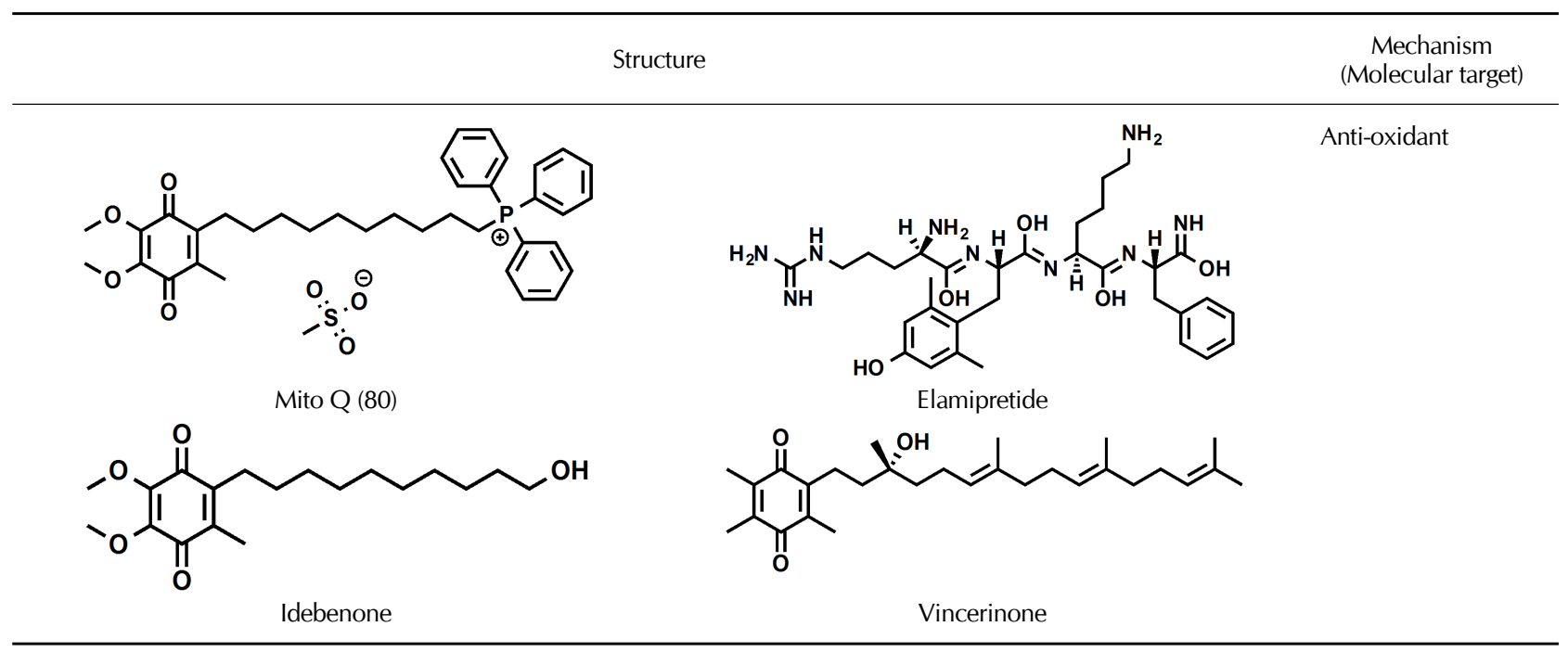

and lowered cerebral $A \beta$ production in addition to restoring overall mitochondrial function (38). Fang et al. also found that mitochondrial dysfunction and defective mitophagy in the brains of $\mathrm{AD}$ patients; but more importantly, when they were treated with chemical inducers of mitophagy, urolithin $A$ and actinonin, cognitive functions of transgenic $A D$ mice and $A D$ nematodes were improved by increasing PINK1 expression, whereas PINK1-deletion nematodes did not show such improvement (39). Urolithin $A$ treatment reduced $A \beta$ and phosphorylated tau proteins in the transgenic AD mice and AD nematodes. A recently completed phase I trial of urolithin A demonstrated a favorable safety profile and significant increases in mitochondrial biomarker genes (40), suggesting that mitophagy-related pathways can provide a promising drug target for neurodegenerative diseases including AD and PD. Currently several small-molecule PINK1 activators have been developed for the treatment of PD, but are still in the preclinical stage because of their poor bioavailability and issues with safety and brain penetration (41). These PINK1 activators are divided into two categories: direct activators that bind to the kinase domain of PINK1 $(42,43)$ and indirect activators that disrupt the mitochondrial membrane potential to initiate PINK1 activation (44). Urolithin A and actinonin were identified via chemical library screening, and they are likely to activate PINK1 via an indirect pathway, although their mechanisms have not been reported.

\section{MITOCHONDRIAL PERMEABILITY TRANSITION PORE (mPTP)}

The mitochondrial permeability transition pore (mPTP) is a multimeric protein complex located in the inner mitochondrial membrane, and the opening of the MPTP induces the loss of mitochondrial membrane potential and structural damage, leading to mitochondrial dysfunction (45). The mitochondrial cyclophilin $\mathrm{D}(\mathrm{CypD})$ is known to be the key component of the mPTP complex, playing a crucial role in the pathogenesis of AD (46-48). Specifically, elevated levels of CypD and $A \beta-C y p D$ complex were found in the cortical mitochondria of the transgenic $\mathrm{AD}$ mice, promoting the opening of the $\mathrm{MPTP}$ and exacerbating neuronal stress and mitochondrial dysfunction (48). Given that CypD is the only validated regulator of the mPTP (49), it has been hypothesized that the suppression of the CypD-mediated mPTP activation can be a novel therapeutic strategy for AD. To test the hypothesis, cyclosporin A (CsA), a well-known inhibitor of CypD, has been extensively studied to examine its potential therapeutic effect in AD. Although CsA inhibits the mPTP opening, CsA is not suitable for clinical application, since it is also an immunosuppressant and cannot penetrate the blood-brain barrier (BBB) effectively. Therefore, research efforts have focused on developing highly specific inhibitors with favorable pharmacokinetics. Elkamhawy et al. developed a library of quinazoline-based compounds that were modified from known cyclophilin A inhibitors, and these compounds demonstrated inhibitory activity against mPTP opening in cell-based assays (50). Valasani et al. reported CypD selective inhibitors, based on molecular docking studies and virtual screening, which also demonstrated the inhibitory effect against $A \beta$-induced mitochondrial dysfunction and cytotoxicity $(51,52)$. Park et al. reported an energy-based pharmacophore model by using the crystal structure of CypD-cyclosporine A (CsA) complex and performed virtual screening to identify novel non-peptidic small-molecule inhibitors of CypD (53). Although $K_{D}$ values determined by surface plasmon resonance analysis indicated that these compounds were not as potent as CsA, it provides new 
insights into the rational design of small-molecule CypD inhibitors for the treatment of AD.

\section{NEUROSTEROIDOGENESIS}

Neuroactive steroids are steroid-based molecules that can rapidly alter the neuronal excitability by interacting with neuronal receptors, such as the $\gamma$-amino-butyric type A (GABAA) receptor (54). Neuroactive steroids can be synthesized de novo inside neurons or from cholesterol transported into mitochondria. In recent years, neuroprotective and neurotrophic effect of neuroactive steroids have been reported, and growing evidence suggests that neuroactive steroids may offer therapeutic opportunities for neurodegenerative diseases including PD and $A D$ (55). In particular, the mitochondrial translocator protein (TSPO) and $17 \beta$-hydroxysteroid dehydrogenase type 10 (17 $\beta$-HSD10) have been studied because of their involvement in mitochondrial dysfunction as well as neurosteroidogenesis. In the following section, we will overview the AD pathogenesis associated with these two target proteins and known chemical ligands for potential therapeutic agents in AD.

\section{TSPO (The translocator protein)}

TSPO is a transmembrane protein located in the outer mitochondrial membrane (OMM). Although the exact role of TSPO still remains to be explained, many studies have suggested that TSPO is associated with the regulation of mitochondrial function, mainly through the cholesterol transport and neuroactive steroid hormone production (56). In AD, TSPO is believed to be linked to two distinct pathological pathways: first, the impaired cholesterol transport because of abnormal expression levels in the damaged neurons (57); second, the activation of the mitochondrial permeability transition pore (mPTP) (58), although the role of TSPO in the mPTP activation is now in question $(59,60)$. Given the regulatory role of TSPO in mitochondrial function and neurotrophic effects, many TSPO ligands have been developed over the past decade. Benzodiazepine compounds, Ro5-4864 and PK 11195, are the most widely used TSPO ligands. Ro5-4864 attenuated the development of AD pathology in the transgenic $\mathrm{AD}$ mice, and the combination therapy using Ro5-4864 and PK 11195 reduced A $\beta$ levels in gonadectomized non-transgenic mice, supporting that TSPO is a potential treatment target for $A D$ (61). More recently, Kim et al. reported a library of TSPO-targeted mitochondrial functional modulators for the treatment of $A D$ (62). These compounds were able to restore mitochondrial function from $A \beta$-induced toxicity and improved cognitive function in transgenic AD mice. Monga et al. also identified two novel TSPO ligands, 2-Cl-MGV-1 and MGV-1, that can prevent LPS-induced activation of microglia. These ligands showed protective effects against ROS generation and exhibited potent anti-inflammatory activity relevant to the neuro-inflammatory diseases (63).
17 $\beta$-hydroxysteroid dehydrogenase type 10 (17 $\beta$-HSD10) $17 \beta$-HSD10, which was referred to as $A \beta$-binding alcohol dehydrogenase (ABAD), is a mitochondrial enzyme associated with the metabolism of steroid hormones. It plays crucial roles in neurosteroidogenesis and isoleucine degradation, and its genetic mutation has been implicated in delayed brain development and brain dysfunction (64). It is an essential enzyme for neuronal survival in a healthy brain, but many studies have also supported that $17 \beta-\mathrm{HSD} 10$ may be a therapeutic target as well as a potential biomarker for $A D$ (65). In particular, increased levels of $17 \beta-$ HSD10 were found in the brains of $A D$ patients and transgenic $A D$ mice (66); the interaction between $A \beta$ and $17 \beta$-HSD10 appeared to promote ROS generation and to induce mitochondrial dysfunction (67). Although the exact mechanism of the specific interactions between $A \beta$ and $17 \beta-$ HSD10 has not been reported yet, research efforts have been made to develop small-molecule inhibitors targeting $17 \beta-\mathrm{HSD} 10$ for the treatment of AD (68). Steroid-based inhibitors demonstrated a highly selective but relatively weak inhibitory effect against the oxidation of allopregnanolone and estradiol (69). Non-steroidal compounds with fused pyrazole (70) and benzothiazole $(71,72)$ showed more potent activity with nanomolar to low micromolar $\mathrm{IC}_{50}$ values. Viswanath et al. reported small-molecule inhibitors based on the receptor-based pharmacophore modeling using the X-ray crystal structure of human $17 \beta-H S D 10$ (73).

\section{OXIDATIVE STRESS}

Mitochondrial oxidative stress is an extensively studied pathological process, since the primary role of mitochondria is ATP production via aerobic cellular respiration. Mitochondria are the largest contributor of cellular reactive oxygen species (ROS), mostly generated by the respiratory chain complexes I and III as byproducts of oxidative phosphorylation (74). In healthy cells, natural antioxidant enzymes, such as superoxide dismutases, glutathione peroxidase, and catalase, swiftly act as scavengers to remove ROS; however, in the brains of $A D$ patients, the catalytic activity of these enzymes is compromised, leading to the accumulation of ROS. An increased level of ROS is particularly detrimental in early-stage $A D$, during which oxidatively damaged proteins and DNA are produced, brain bioenergetics are impaired, and the structural and functional integrity of neuronal cells is severely altered (75, 76). Moreover, recent studies suggest that redox-active metals such as copper and zinc can promote $A \beta$ aggregation and ROS generation depending on their oxidation states, further contributing to the progress of $\mathrm{AD}$ (77). Administration of exogeneous anti-oxidants is considered to be a promising strategy to block the ROS-induced damage and prevent further disease progression. Natural small-molecule anti-oxidants, including coenzyme Q (78) and vitamins C and E (79), were the first tested compounds among many other potential therapeutic anti-oxidants, although these naturally derived 
molecules were not effective in clinical trials because they could not penetrate the BBB or localize into mitochondria. To improve mitochondrial localization, mitochondria-targeted antioxidants, such as mito Q (80) and mitoVit E (81), have been developed. These molecules contain coenzyme $\mathrm{Q}$ and vitamin $\mathrm{E}$, but also have an additional triphenylphosphonium group, a lipophilic cation that can localize to the negatively charged mitochondrial membrane. Similarly, various peptidic anti-oxidants, including elamipretide, XJB peptides, and mito-glutathione, as well as coenzyme $\mathrm{Q}$ analogs idebenone and vincerinone, have been developed to improve cellular penetration and mitochondrial localization $(82,83)$. These compounds demonstrated excellent mitochondrial localization and anti-oxidant activity in vitro, and some were advanced to clinical trials for various mitochondrial diseases (84). Although these agents were mostly ineffective in clinical trials for $A D$, their proven anti-oxidant effects and favorable safety profile can be advantageous features for the development of preventive or combination therapy in the future.

\section{CONCLUSION}

In this mini review, we outlined the mitochondrial pathways that are directly associated with $\mathrm{AD}$ pathology and surveyed their small-molecule modulators for potential therapeutic applications. Compounds linked to mitochondrial quality control, such as division inhibitors and mitophagy activators, are relative newcomers in the field, demonstrating improved mitochondrial function and cognitive enhancement in animal models of AD. Although their clinical efficacy and safety must be examined, their mode of action is particularly intriguing, since these compounds can boost critical mitochondrial function in damaged cells. Another potential therapeutic strategy is the suppression of the mPTP to restore the structural and functional integrity of mitochondria in AD neurons. CypD is the most well-characterized component of the MPTP, and chemical modulation of CypD blocked the formation of the $\mathrm{mPTP}$ and restored mitochondrial function in animal models of $A D$. CypD has a highly conserved structure with other cyclophilins that are abundant and ubiquitous, thus specific modulation of the mitochondrial CypD is probably the key to the successful clinical development.

Compounds promoting neurosteroidogenesis and ROSscavenging anti-oxidants have been extensively studied in many clinical studies as well as animal models because of their general protective effects against inflammation and oxidative stress. Interestingly, the protein structures and cellular locations of the potential targets involved in neurosteroidogenesis, TSPO and 17 $\beta-\mathrm{HSD} 10$, have been recently elucidated, and new generations of chemical modulators have been introduced, which may enable to generate highly potent and selective therapeutic agents for AD in the near future. On the other hand, ROS scavengers have proven their anti-oxidant activity and safety, already being sold in the market or in the later stage of clinical trials. Recent developments in the ROS-scavenger studies are mostly focused on the targeted delivery of anti-oxidants to improve bioavailability and efficacy for various mitochondrial diseases. Mounting evidence suggests that mitochondrial function is associated with diverse diseases, including neurodegenerative diseases, metabolic disorders, and cancers; hence mitochondria-specific delivery of therapeutic agents can be an alternative to using existing drugs.

Identifying new therapeutic candidates for $A D$ is still an enormously challenging task, because of the lack of reliable biomarkers, and the difficulties in clinical translation of animal models to human pathology, particularly given the long-term progression of the disease. In addition, tissue-specificity and brain penetration of the potential therapeutic agents pose another obstacle to overcome for effective AD therapy. Considering the multifactorial nature of $A D$, tackling a single target alone may not result in beneficial therapeutic outcomes, as we have seen in many previous clinical trials of AD. Compounds that can restore mitochondrial function not only have the potential to slow down the progress of the disease, but also can provide an additional therapeutic option in combination with currently available therapy.

\section{ACKNOWLEDGEMENTS}

This work was supported by the National Research Council of Science \& Technology grant by the Korea government (MSIP) (No.CRC-15-04-KIST). J. Lee was supported by the Sungshin Women's University Research Grant of 2019-1-82-015.

\section{CONFLICTS OF INTEREST}

The authors have no conflicting interests.

\section{REFERENCES}

1. Anand R, Gill KD and Mahdi AA (2014) Therapeutics of Alzheimer's disease: Past, present and future. Neuropharmacology $76 \mathrm{Pt} A, 27-50$

2. Perry G, Nunomura A, Hirai K, Takeda A, Aliev G and Smith MA (2000) Oxidative damage in Alzheimer's disease: the metabolic dimension. Int J Dev Neurosci 18, 417-421

3. Schmitt K, Grimm A, Kazmierczak A, Strosznajder JB, Gotz J and Eckert A (2012) Insights into mitochondrial dysfunction: aging, amyloid-beta, and tau-A deleterious trio. Antioxid Redox Signal 16, 1456-1466

4. Yao J, Irwin RW, Zhao L, Nilsen J, Hamilton RT and Brinton RD (2009) Mitochondrial bioenergetic deficit precedes Alzheimer's pathology in female mouse model of Alzheimer's disease. Proc Natl Acad Sci U S A 106, 14670-14675

5. Swerdlow RH, Burns JM and Khan SM (2010) The Alzheimer's disease mitochondrial cascade hypothesis. J Alzheimers Dis 20 Suppl 2, S265-279 
6. Hauptmann S, Scherping I, Drose S et al (2009) Mitochondrial dysfunction: an early event in Alzheimer pathology accumulates with age in $A D$ transgenic mice. Neurobiol Aging 30, 1574-1586

7. Shulman RG, Rothman DL, Behar KL and Hyder F (2004) Energetic basis of brain activity: implications for neuroimaging. Trends Neurosci 27, 489-495

8. Mao P and Reddy PH (2011) Aging and amyloid beta-induced oxidative DNA damage and mitochondrial dysfunction in Alzheimer's disease: implications for early intervention and therapeutics. Biochim Biophys Acta $1812,1359-1370$

9. Keil U, Bonert A, Marques CA et al (2004) Amyloid beta-induced changes in nitric oxide production and mitochondrial activity lead to apoptosis. J Biol Chem 279, 50310-50320

10. Eckert GP, Renner K, Eckert SH et al (2012) Mitochondrial dysfunction-a pharmacological target in Alzheimer's disease. Mol Neurobiol 46, 136-150

11. Johri A and Beal MF (2012) Mitochondrial dysfunction in neurodegenerative diseases. J Pharmacol Exp Ther 342, 619-630

12. Moreira PI, Carvalho C, Zhu X, Smith MA and Perry G (2010) Mitochondrial dysfunction is a trigger of Alzheimer's disease pathophysiology. Biochim Biophys Acta 1802, 210

13. Müller WE, Eckert A, Kurz C, Eckert GP and Leuner K (2010) Mitochondrial dysfunction: common final pathway in brain aging and Alzheimer's disease-therapeutic aspects. Mol Neurobiol 41, 159-171

14. Swerdlow RH, Burns JM and Khan SM (2014) The Alzheimer's disease mitochondrial cascade hypothesis: progress and perspectives. Biochim Biophys Acta 1842, 1219-1231

15. Farlow MR, Miller ML and Pejovic V (2008) Treatment options in Alzheimer's disease: maximizing benefit, managing expectations. Dement Geriatr Cogn Disord 25, 408-422

16. Moehle EA, Shen K and Dillin A (2019) Mitochondrial proteostasis in the context of cellular and organismal health and aging. J Biol Chem 294, 5396-5407

17. Tatsuta $T$ and Langer $T$ (2008) Quality control of mitochondria: protection against neurodegeneration and ageing. EMBO J 27, 306-314

18. Franco-lborra S, Vila M and Perier C (2018) Mitochondrial Quality Control in Neurodegenerative Diseases: Focus on Parkinson's Disease and Huntington's Disease. Front Neurosci 12, 342

19. Tilokani L, Nagashima S, Paupe V and Prudent J (2018) Mitochondrial dynamics: overview of molecular mechanisms. Essays Biochem 62, 341-360

20. Burte F, Carelli V, Chinnery PF and Yu-Wai-Man P (2015) Disturbed mitochondrial dynamics and neurodegenerative disorders. Nat Rev Neurol 11, 11-24

21. Kandimalla R and Reddy PH (2016) Multiple faces of dynamin-related protein 1 and its role in Alzheimer's disease pathogenesis. Biochim Biophys Acta 1862, 814-828

22. Reddy PH and Beal MF (2008) Amyloid beta, mitochondrial dysfunction and synaptic damage: implications for cognitive decline in aging and Alzheimer's disease. Trends Mol Med 14, 45-53

23. Manczak M and Reddy PH (2012) Abnormal interaction between the mitochondrial fission protein Drp1 and hyperphosphorylated tau in Alzheimer's disease neurons: implications for mitochondrial dysfunction and neuronal damage. Hum Mol Genet 21, 2538-2547

24. Silva DF, Selfridge JE, Lu J et al (2013) Bioenergetic flux, mitochondrial mass and mitochondrial morphology dynamics in $\mathrm{AD}$ and $\mathrm{MCl}$ cybrid cell lines. Hum Mol Genet 22, 3931-3946

25. Calkins MJ, Manczak M, Mao P, Shirendeb $U$ and Reddy $\mathrm{PH}$ (2011) Impaired mitochondrial biogenesis, defective axonal transport of mitochondria, abnormal mitochondrial dynamics and synaptic degeneration in a mouse model of Alzheimer's disease. Hum Mol Genet 20, 4515-4529

26. Manczak M, Calkins MJ and Reddy PH (2011) Impaired mitochondrial dynamics and abnormal interaction of amyloid beta with mitochondrial protein Drp1 in neurons from patients with Alzheimer's disease: implications for neuronal damage. Hum Mol Genet 20, 2495-2509

27. Cassidy-Stone A, Chipuk JE, Ingerman E et al (2008) Chemical inhibition of the mitochondrial division dynamin reveals its role in Bax/Bak-dependent mitochondrial outer membrane permeabilization. Dev Cell 14, 193-204

28. Wang W, Yin J, Ma X et al (2017) Inhibition of mitochondrial fragmentation protects against Alzheimer's disease in rodent model. Hum Mol Genet 26, 4118-4131

29. Baek SH, Park SJ, Jeong JI et al (2017) Inhibition of Drp1 Ameliorates Synaptic Depression, Abeta Deposition, and Cognitive Impairment in an Alzheimer's Disease Model. J Neurosci 37, 5099-5110

30. Guo X, Disatnik MH, Monbureau $M$, Shamloo $M$, Mochly-Rosen D and Qi X (2013) Inhibition of mitochondrial fragmentation diminishes Huntington's disease-associated neurodegeneration. J Clin Invest 123, 5371-5388

31. Qi X, Qvit N, Su YC and Mochly-Rosen D (2013) A novel Drp1 inhibitor diminishes aberrant mitochondrial fission and neurotoxicity. J Cell Sci 126, 789-802

32. Numadate A, Mita $Y$, Matsumoto $Y$, Fujii $S$ and Hashimoto Y (2014) Development of 2-thioxoquinazoline-4-one derivatives as dual and selective inhibitors of dynaminrelated protein 1 (Drp1) and puromycin-sensitive aminopeptidase (PSA). Chem Pharm Bull (Tokyo) 62, 979-988

33. Mallat A, Uchiyama LF, Lewis SC et al (2018) Discovery and characterization of selective small molecule inhibitors of the mammalian mitochondrial division dynamin, DRP1. Biochem Biophys Res Commun 499, 556-562

34. Kuruva CS, Manczak M, Yin X, Ogunmokun G, Reddy AP and Reddy PH (2017) Aqua-soluble DDQ reduces the levels of Drp1 and Abeta and inhibits abnormal interactions between Abeta and Drp1 and protects Alzheimer's disease neurons from Abeta- and Drp1-induced mitochondrial and synaptic toxicities. Hum Mol Genet 26, 3375-3395

35. Smith G and Gallo G (2017) To mdivi-1 or not to mdivi-1: Is that the question? Dev Neurobiol 77, 1260-1268

36. Valente EM, Abou-Sleiman PM, Caputo V et al (2004) Hereditary early-onset Parkinson's disease caused by mutations in PINK1. Science 304, 1158-1160

37. Pickrell AM and Youle RJ (2015) The roles of PINK1, 
parkin, and mitochondrial fidelity in Parkinson's disease. Neuron 85, 257-273

38. Du F, Yu Q, Yan S et al (2017) PINK1 signalling rescues amyloid pathology and mitochondrial dysfunction in Alzheimer's disease. Brain 140, 3233-3251

39. Fang EF, Hou Y, Palikaras K et al (2019) Mitophagy inhibits amyloid-beta and tau pathology and reverses cognitive deficits in models of Alzheimer's disease. Nat Neurosci 22, 401-412

40. Andreux PA, Blanco-Bose W, Ryu D et al (2019) The mitophagy activator urolithin $A$ is safe and induces a molecular signature of improved mitochondrial and cellular health in humans. Nat Metab 1, 595

41. Lambourne OA and Mehellou Y (2018) Chemical Strategies for Activating PINK1, a Protein Kinase Mutated in Parkinson's Disease. Chembiochem 19, 2433-2437

42. Hertz NT, Berthet A, Sos ML et al (2013) A neo-substrate that amplifies catalytic activity of parkinson's-diseaserelated kinase PINK1. Cell 154, 737-747

43. Osgerby L, Lai YC, Thornton PJ et al (2017) Kinetin Riboside and Its ProTides Activate the Parkinson's Disease Associated PTEN-Induced Putative Kinase 1 (PINK1) Independent of Mitochondrial Depolarization. J Med Chem 60, 3518-3524

44. Barini E, Miccoli A, Tinarelli F et al (2018) The Anthelmintic Drug Niclosamide and Its Analogues Activate the Parkinson's Disease Associated Protein Kinase PINK1. Chembiochem 19, 425-429

45. Halestrap AP (2009) What is the mitochondrial permeability transition pore? J Mol Cell Cardiol 46, 821-831

46. Baines CP, Kaiser RA, Purcell NH et al (2005) Loss of cyclophilin $\mathrm{D}$ reveals a critical role for mitochondrial permeability transition in cell death. Nature 434, 658-662

47. Du H, Guo L, Fang F et al (2008) Cyclophilin D deficiency attenuates mitochondrial and neuronal perturbation and ameliorates learning and memory in Alzheimer's disease. Nat Med 14, 1097-1105

48. Du H, Guo L, Zhang W, Rydzewska M and Yan S (2011) Cyclophilin D deficiency improves mitochondrial function and learning/memory in aging Alzheimer disease mouse model. Neurobiol Aging 32, 398-406

49. Briston T, Selwood DL, Szabadkai G and Duchen MR (2019) Mitochondrial Permeability Transition: A Molecular Lesion with Multiple Drug Targets. Trends Pharmacol Sci 40, 50-70

50. Elkamhawy A, Lee J, Park BG, Park I, Pae AN and Roh EJ (2014) Novel quinazoline-urea analogues as modulators for Abeta-induced mitochondrial dysfunction: design, synthesis, and molecular docking study. Eur J Med Chem 84, 466-475

51. Valasani KR, Sun Q, Fang D et al (2016) Identification of a Small Molecule Cyclophilin D Inhibitor for Rescuing Abeta-Mediated Mitochondrial Dysfunction. ACS Med Chem Lett 7, 294-299

52. Valasani KR, Vangavaragu JR, Day VW and Yan SS (2014) Structure based design, synthesis, pharmacophore modeling, virtual screening, and molecular docking studies for identification of novel cyclophilin D inhibitors. J Chem Inf Model 54, 902-912

53. Park I, Londhe AM, Lim JW et al (2017) Discovery of non-peptidic small molecule inhibitors of cyclophilin D as neuroprotective agents in Abeta-induced mitochondrial dysfunction. J Comput Aided Mol Des 31, 929-941

54. Paul SM and Purdy RH (1992) Neuroactive steroids. FASEB J 6, 2311-2322

55. Porcu P, Barron AM, Frye CA et al (2016) Neurosteroidogenesis Today: Novel Targets for Neuroactive Steroid Synthesis and Action and Their Relevance for Translational Research. J Neuroendocrinol 28, 12351

56. Rupprecht R, Papadopoulos V, Rammes G et al (2010) Translocator protein (18 kDa) (TSPO) as a therapeutic target for neurological and psychiatric disorders. Nat Rev Drug Discov 9, 971-988

57. Banati RB, Middleton RJ, Chan R et al (2014) Positron emission tomography and functional characterization of a complete PBR/TSPO knockout. Nat Commun 5, 5452

58. Li J, Wang J and Zeng Y (2007) Peripheral benzodiazepine receptor ligand, PK11195 induces mitochondria cytochrome c release and dissipation of mitochondria potential via induction of mitochondria permeability transition. Eur J Pharmacol 560, 117-122

59. Sileikyte J, Blachly-Dyson E, Sewell R et al (2014) Regulation of the mitochondrial permeability transition pore by the outer membrane does not involve the peripheral benzodiazepine receptor (Translocator Protein of $18 \mathrm{kDa}$ (TSPO)). J Biol Chem 289, 13769-13781

60. Selvaraj $V$ and Stocco DM (2015) The changing landscape in translocator protein (TSPO) function. Trends Endocrinol Metab 26, 341-348

61. Barron AM, Garcia-Segura LM, Caruso D et al (2013) Ligand for translocator protein reverses pathology in a mouse model of Alzheimer's disease. J Neurosci 33, 8891-8897

62. Kim T, Yang HY, Park BG et al (2017) Discovery of benzimidazole derivatives as modulators of mitochondrial function: A potential treatment for Alzheimer's disease. Eur J Med Chem 125, 1172-1192

63. Monga S, Nagler R, Amara R, Weizman A and Gavish M (2019) Inhibitory Effects of the Two Novel TSPO Ligands 2-Cl-MGV-1 and MGV-1 on LPS-induced Microglial Activation. Cells 8, 486

64. Yang SY, He XY and Miller D (2011) Hydroxysteroid (17beta) dehydrogenase $X$ in human health and disease. Mol Cell Endocrinol 343, 1-6

65. Yang SY, He XY, Isaacs C, Dobkin C, Miller D and Philipp M (2014) Roles of 17beta-hydroxysteroid dehydrogenase type 10 in neurodegenerative disorders. J Steroid Biochem Mol Biol 143, 460-472

66. He XY, Wegiel J and Yang SY (2005) Intracellular oxidation of allopregnanolone by human brain type 10 17beta-hydroxysteroid dehydrogenase. Brain Res 1040, 29-35

67. Lustbader JW, Cirilli M, Lin C et al (2004) ABAD directly links Abeta to mitochondrial toxicity in Alzheimer's disease. Science 304, 448-452

68. Morsy A and Trippier PC (2019) Amyloid-Binding Alcohol Dehydrogenase (ABAD) Inhibitors for the Treatment of Alzheimer's Disease. J Med Chem 62, 4252-4264

69. Boutin S, Roy J, Maltais R, Alata W, Calon F and Poirier D (2018) Identification of steroidal derivatives inhibiting the 
transformations of allopregnanolone and estradiol by 17beta-hydroxysteroid dehydrogenase type 10. Bioorg Med Chem Lett 28, 3554-3559

70. Lim YA, Grimm A, Giese M et al (2011) Inhibition of the mitochondrial enzyme $A B A D$ restores the amyloid-betamediated deregulation of estradiol. PLoS One 6, e28887

71. Valasani KR, Hu G, Chaney MO and Yan SS (2013) Structure-based design and synthesis of benzothiazole phosphonate analogues with inhibitors of human ABAD-Abeta for treatment of Alzheimer's disease. Chem Biol Drug Des 81, 238-249

72. Benek O, Hroch L, Aitken L et al (2017) 6-benzothiazolyl ureas, thioureas and guanidines are potent inhibitors of ABAD/17beta-HSD10 and potential drugs for Alzheimer's disease treatment: Design, synthesis and in vitro evaluation. Med Chem 13, 345-358

73. Viswanath ANI, Kim T, Jung SY, Lim SM and Pae AN (2017) In silico-designed novel non-peptidic ABAD LD hot spot mimetics reverse Abeta-induced mitochondrial impairments in vitro. Chem Biol Drug Des 90, 1041-1055

74. Holmstrom KM and Finkel T (2014) Cellular mechanisms and physiological consequences of redox-dependent signalling. Nat Rev Mol Cell Biol 15, 411-421

75. Tonnies E and Trushina E (2017) Oxidative Stress, Synaptic Dysfunction, and Alzheimer's Disease. J Alzheimers Dis 57, 1105-1121

76. Cheignon $C$, Tomas $M$, Bonnefont-Rousselot $D$, Faller $P$, Hureau C and Collin F (2018) Oxidative stress and the amyloid beta peptide in Alzheimer's disease. Redox Biol $14,450-464$
77. Barnham KJ and Bush Al (2014) Biological metals and metal-targeting compounds in major neurodegenerative diseases. Chem Soc Rev 43, 6727-6749

78. Dumont M, Kipiani K, Yu F et al (2011) Coenzyme Q10 decreases amyloid pathology and improves behavior in a transgenic mouse model of Alzheimer's disease. J Alzheimers Dis 27, 211-223

79. Gugliandolo A, Bramanti P and Mazzon E (2017) Role of Vitamin $E$ in the Treatment of Alzheimer's Disease: Evidence from Animal Models. Int J Mol Sci 18, 2504

80. McManus MJ, Murphy MP and Franklin JL (2011) The mitochondria-targeted antioxidant MitoQ prevents loss of spatial memory retention and early neuropathology in a transgenic mouse model of Alzheimer's disease. J Neurosci 31, 15703-15715

81. Covey MV, Murphy MP, Hobbs CE, Smith RA and Oorschot DE (2006) Effect of the mitochondrial antioxidant, Mito Vitamin E, on hypoxic-ischemic striatal injury in neonatal rats: a dose-response and stereological study. Exp Neurol 199, 513-519

82. Webb M, Sideris DP and Biddle M (2019) Modulation of mitochondrial dysfunction for treatment of disease. Bioorg Med Chem Lett 29, 1270-1277

83. Oliver DMA and Reddy PH (2019) Small molecules as therapeutic drugs for Alzheimer's disease. Mol Cell Neurosci 96, 47-62

84. El-Hattab AW, Zarante AM, Almannai M and Scaglia F (2017) Therapies for mitochondrial diseases and current clinical trials. Mol Genet Metab 122, 1-9 